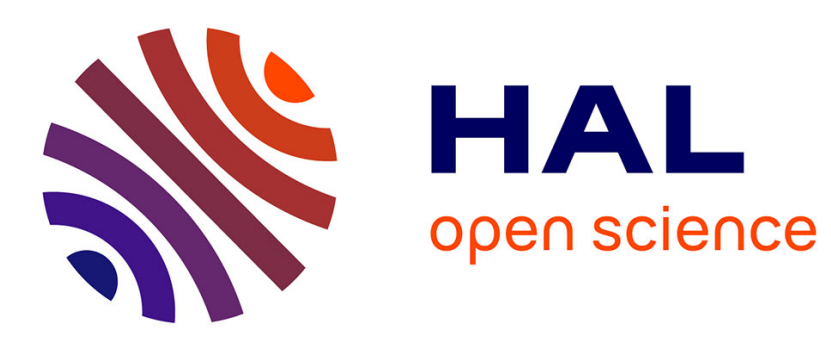

\title{
Eddy-current non-destructive testing system for the determination of crack orientation
}

Rimond Hamia, Christophe Cordier, Christophe Dolabdjian

\section{To cite this version:}

Rimond Hamia, Christophe Cordier, Christophe Dolabdjian. Eddy-current non-destructive testing system for the determination of crack orientation. NDT \& E International, 2014, 61, pp.24-28. 10.1016/j.ndteint.2013.09.005 . hal-00987058

\section{HAL Id: hal-00987058 \\ https://hal.science/hal-00987058}

Submitted on 5 May 2014

HAL is a multi-disciplinary open access archive for the deposit and dissemination of scientific research documents, whether they are published or not. The documents may come from teaching and research institutions in France or abroad, or from public or private research centers.
L'archive ouverte pluridisciplinaire $\mathbf{H A L}$, est destinée au dépôt et à la diffusion de documents scientifiques de niveau recherche, publiés ou non, émanant des établissements d'enseignement et de recherche français ou étrangers, des laboratoires publics ou privés. 


\title{
Eddy-current non-destructive testing system for the determination of crack orientation
}

\author{
Rimond Hamia, Christophe Cordier *, Christophe Dolabdjian \\ GREYC (CNRS-UMR6072), Université de Caen Basse Normandie E ENSICAEN, 6 Bd. Marechal Juin, 14050 Caen, France
}

\begin{abstract}
A B S T R A C T
Detecting the orientation of deep oriented cracks is a major challenge in the development of EddyCurrent (EC) Non-Destructive Testing (NDT). In fact, the detection sensitivity of EC-NDT depends on the interaction between the crack length direction and the EC flowing in the materials. In conventional EC-NDT systems, the induced currents are primarily generated along a single direction in the tested sample. This paper presents a new excitation method for generating a pseudo-rotating ac magnetic field and, consequently, pseudo-rotating eddy currents. This method significantly improves the detection of deep cracks of any orientation. The detected signal due to the crack is measured by an Improved Giant Magneto-Resistance Magnetometer (IGMRM). The magnetic flux density signature of the crack is studied using a 3D finite element model. Numerical and experimental results demonstrate the potential and the efficiency of this method.
\end{abstract}

\section{Introduction}

The detection of deep cracks in structures and materials is an important issue for EC-NDT systems. The detection depends not only on the sensitivity of the magnetometer but also on the interaction between the cracks and the EC flowing in the tested structures. For example, the NDT-EC systems developed in [1] has a preferential direction for detecting cracks. In fact, the inducer was directed to induce a current perpendicular to the crack length. As an example, Fig. 1 shows an aluminum sample where the EC flow is generated by a single wire as an inducer that is oriented along the $z$ axis. In the two given configurations, the crack is either perpendicular or parallel to the EC. When the EC flow is applied perpendicular to the crack length, as shown in Fig. 1a, a large flux disturbance appears near the defect. However, when a crack or another anomaly is parallel to the EC flow, a small flux disturbance occurs and the possibility to sense the defect decreases significantly (see Fig. 1b).

Most of the developed EC-NDT systems use inducers that create a uniform distribution of the EC in the tested structure [1-5]. In the papers describing these systems, the cracks are almost always oriented to maximize the interaction with the EC flow. In the real world of NDT, neither the existence nor the orientations of the defects are known. Thus, the optima detecting configuration cannot be set a priori. To overcome this problem, systems using a rotational GMR-EC magnetometer [6] or a SQUID probe [7], were

\footnotetext{
* Corresponding author. Tel.: +33231452690.

E-mail address: christophe.cordier@unicaen.fr (C. Cordier).
}

built with the capability of detecting cracks of any orientation. In this study, we propose an original and simple EC system. The system exhibits detection performances that are independent of the orientation of the cracks. The system incorporates a specific inducer that creates a rotating magnetic flux density without the mechanical rotation of the entire testing system. An IMGMR magnetometer is used as a sensing element $[8,9]$. The benefits of such an excitation method are to improve the detection sensitivity of the EC system and to simplify the characterization of the cracks (in particular, their orientation).

We have used a 3D numerical model based on the Finite Element Method (FEM) to simulate the NDT inspection process and to predict the corresponding signal sensed by the IGMR magnetometer. This numerical model has been previously validated by comparing the numerical and experimental results obtained for the detection of deep cracks in a benchmark sample [6]. The rotating magnetic field inducer is described in section 2. The contribution for oriented crack detection is given in sections 3 and 4 . Section 5 is devoted to experimental validation and is followed by a conclusion.

\section{Rotating magnetic field inducer}

As we mentioned in section 1, the strength of the magnetic field signal due to defects depends on the orientation of the cracks with respect to the EC flow direction in the sample. To make the detection sensitivity of the EC system independent of the orientation of the cracks, we use an inducer generating a rotating magnetic flux density. This rotating type of excitation is difficult to achieve with conventional circular coil inducers. The proposed 

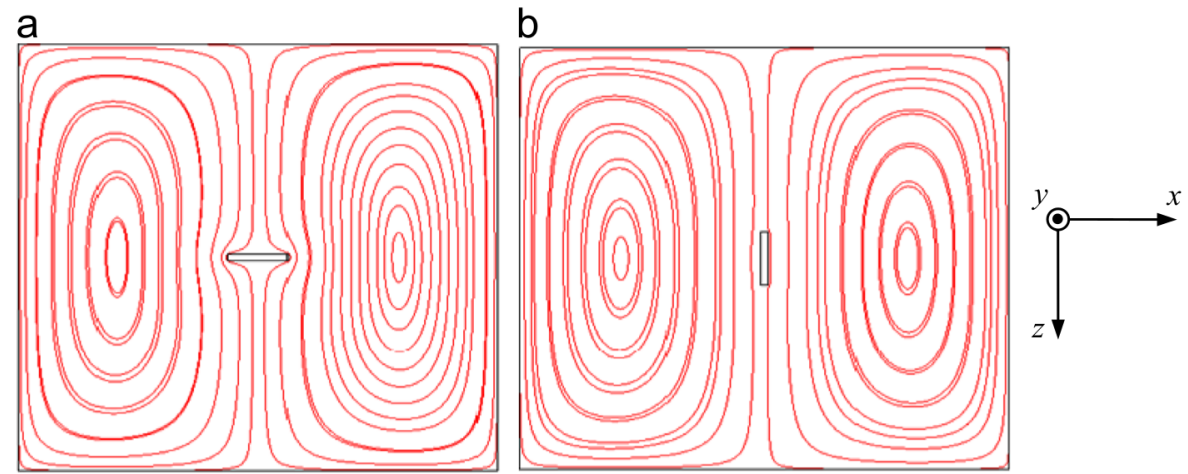

Fig. 1. Eddy current flow distributions around a crack oriented along the a) $x$ axis and b) $z$ axis.
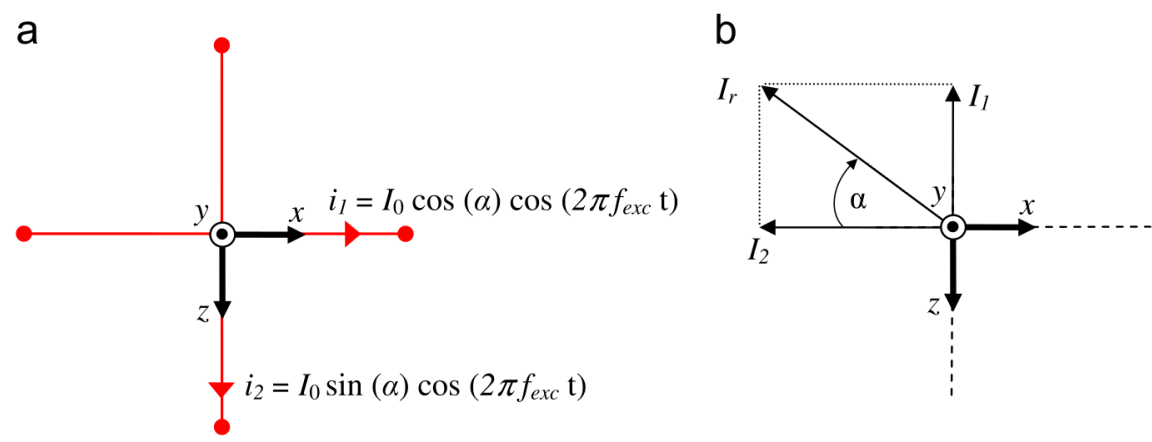

Fig. 2. (a) Pseudo-rotating magnetic field inducer, (b) Resultant Eddy Current, $I_{r}$, in the sample above the intersection of the inducers.

inducer consists of two orthogonal wires fed by alternating currents, whose amplitudes are modulated to create EC flow in a controlled direction within the inspected areas. The currents in the two wires are sinusoidal and have the same frequency and phase. For non-destructive system inspection scanning the $(x O z)$ plane, we used wires oriented along the $x$ - and $z$-axis. The excitation current expressions are given by

$i_{1}=I_{0} \cos (\alpha) \cos \left(2 \pi f_{\text {exc }} t\right)$ for the wire along the $x$ axis

$i_{2}=I_{0} \sin (\alpha) \cos \left(2 \pi f_{\text {exc }} t\right)$ for the wire along the $z$ axis

where $f_{\text {exc }}, I_{0}$ and $\alpha$ are the excitation frequency, the maximum amplitude and the excitation angle, respectively.

Each of the two wires creates eddy currents. According to Lenz's law, the eddy currents are oriented in the opposite direction of the currents which gave rise to them. The combination of these two EC flows produces a current, $I_{r}$, which have the same frequency, $f_{\text {exc }}$. It yields

$I_{r}=I_{1}+I_{2}$

where $I_{1}$ and $I_{2}$ are the eddy currents induced by the inductors oriented along the $x$ - and $z$-axis, respectively.

Below the intersection of the inducer, the total current, $I_{r}$, has an orientation that depends on the angle $\alpha$ with respect to the $x$ axis, as detailed in Fig. 2. By varying the excitation angle $\alpha$, we are able to control the direction of the EC flow in an area below the inducer intersection. The rotation of the induced currents was verified by modeling this cross inducer above an aluminum plate. The eddy current distributions obtained using FEM simulations are shown in Fig. 3 for different values of $\alpha$ in the range of $0^{\circ}-180^{\circ}$. The current distributions for angles between $180^{\circ}$ and $360^{\circ}$ are symmetric to those between $0^{\circ}$ and $180^{\circ}$.

These simulations clearly demonstrate that the EC flow rotates in the $(x O z)$ plane with the excitation angle $\alpha$. By using this type of inducer, the best detection configuration can be selected regardless of the orientation of the crack. Furthermore, the variation of the signal amplitude due to the crack versus the direction of the current flow can provide information about the geometry of the crack and especially its direction.

\section{Numerical analysis}

The purpose of this section is to assess the most advantageous configuration of the inspection system for the detection of deep oriented cracks. FEM simulations were performed on two aluminum samples, one containing no defect and the other containing a single crack centered and oriented in the direction at an angle denoted $\beta$ with respect to the $x$-axis (Fig. 4). The sensor was set above the inducer and vertically at the intersection of the two wires.

The simulations were performed with and without a crack to evaluate the highest signal induced by the crack, $\left|\Delta B_{i}\right|$. In particular, we studied the variations of $\left|\Delta B_{i}\right|$ versus the excitation angle $\alpha$ and versus the position $x$ of the system relative to the center of the sample. The magnetic signature of the crack is then expressed by

$$
\left|\Delta B_{i}(x, \alpha)\right|=\left|B_{i, \operatorname{crack}}(x, \alpha)-B_{i, 0}(x)\right|
$$

and

$\left|\Delta B_{i}\right|_{\text {max }}=\max \left|\Delta B_{i}(x, \alpha)\right|$

where, $B_{x, \text { crack }}$ and $B_{x, 0}$ are the magnetic flux density, sensed along the $x$-axis, at the magnetometer location, with and without crack, respectively. The calculations were performed for two orthogonal orientations of the crack as

- crack oriented along the $x$-axis $\left(\beta=0^{\circ}\right)$ parallel to the scan direction,

- crack oriented along the $z$-axis $\left(\beta=90^{\circ}\right)$ perpendicular to the scan direction. 

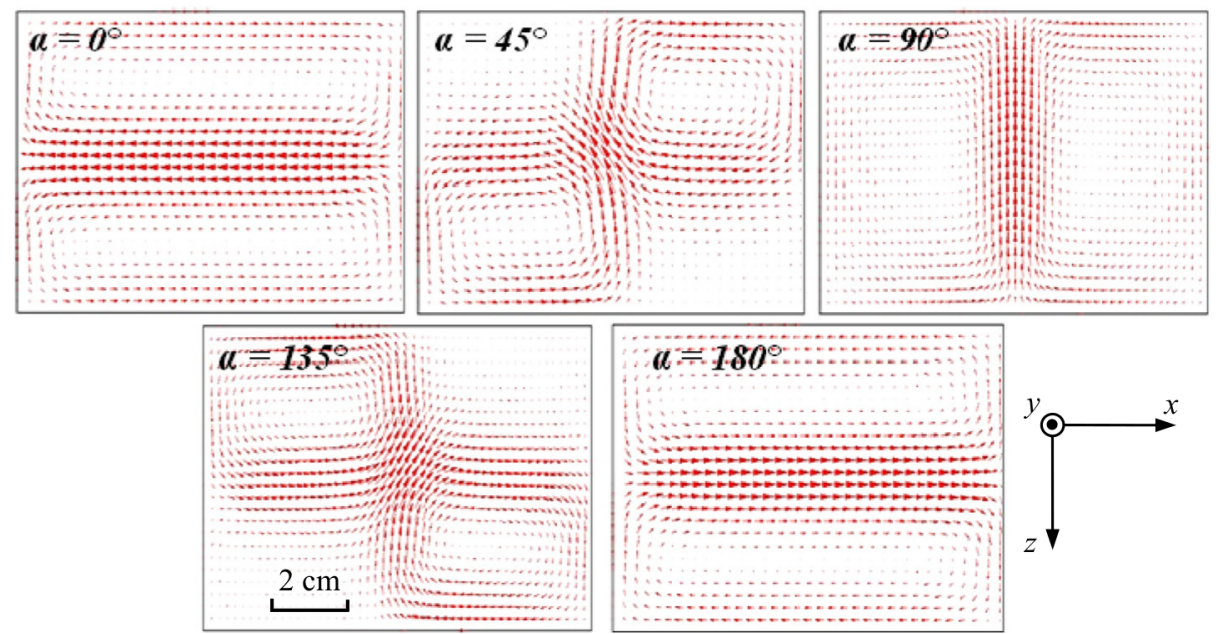

Fig. 3. Distribution of the EC flow in an aluminum plate for different values of the excitation angle $\alpha\left(\sigma \approx 1.68 \times 10^{7} \mathrm{~S} / \mathrm{m}, f_{\text {exc }}=400 \mathrm{~Hz}\right)$. The inducer lift-off is $1 \mathrm{~mm}$.

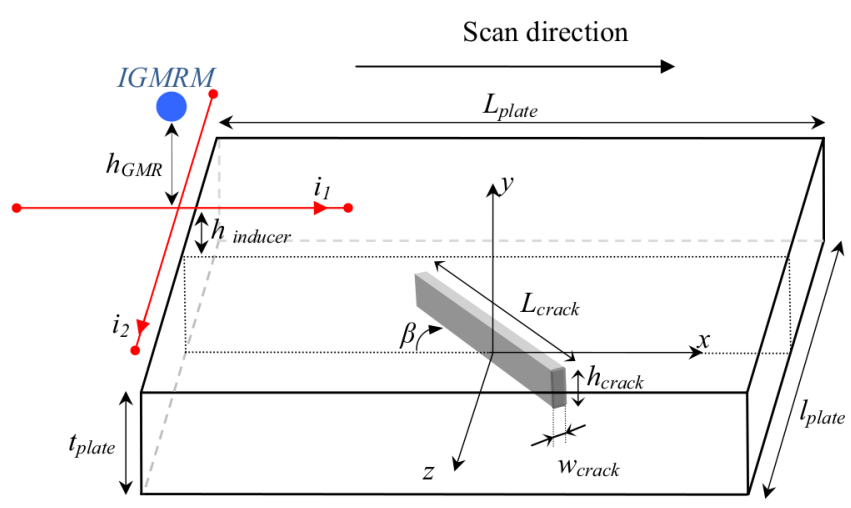

Fig. 4. Sketch view of the EC-NDT system and the tested aluminum sample $\left(L_{\text {plate }}=160 \mathrm{~mm}, \quad l_{\text {plate }}=50 \mathrm{~mm}, \quad t_{\text {plate }}=10 \mathrm{~mm}, \quad h_{\text {inducer }}=1 \mathrm{~mm}, \quad h_{\mathrm{GMR}}=0.5 \mathrm{~mm}\right.$, $\left.L_{\text {crack }}=8 \mathrm{~mm}, h_{\text {crack }}=8 \mathrm{~mm}, w_{\text {crack }}=0.1 \mathrm{~mm}, \sigma \approx 1.68 \times 10^{7} \mathrm{~S} / \mathrm{m}, f_{\text {exc }}=400 \mathrm{~Hz}\right)$.

\section{Table 1}

Optimal detection parameters for each component of the magnetic flux density and for the two crack orientations $\left(\beta=0^{\circ}\right.$ and $\left.90^{\circ}\right)$. The current line distribution around the crack is represented in each optimal configuration. The magnetic flux density is expressed in Tesla per ampere, as it has been normalized by $I_{0}$.

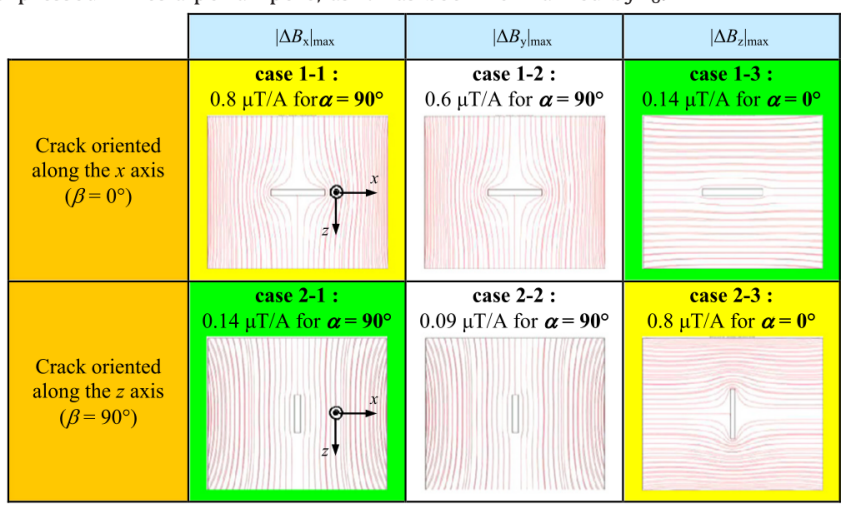

For each direction of the crack, the sensor was positioned to sense the three magnetic field components along the reference axis $(O x y z)$. For each of the six configurations, we determined the configuration that improves the detection and provides relevant information about the orientation of the detected crack.
The results are summarized in Table 1 . We determined for the selected component $i$ and the selected crack orientation, the maximum field amplitude $\left|\Delta B_{i}\right|_{\max }$ and the angle $\alpha$ which lead to this maximum. Moreover, the current line distributions are presented in each optimal configuration.

Table 1 indicates that the measurement of $B_{x}$ and $B_{z}$ is more advantageous for the detection of the crack oriented along the $x$ axis and $z$ axis (cases 1-1 and 2-3), respectively, because in these cases the signal amplitude of the defect is highest. Furthermore, the results demonstrate that for some components of the magnetic field and particular orientations of the crack, the signal $\left|\Delta B_{i}\right|$ reaches a maximum when the EC flows are parallel to the crack length (cases 1-3, 2-1 and 2-2). Nevertheless, if we consider a particular magnetic flux density component $i$, the highest amplitude signature, $\left|\Delta B_{i}\right|_{\max }$, is obtained when the EC flow is perpendicular to the crack direction. This configuration corresponds to the maximal interaction between the defect and the EC.

Additionally, note that for an excitation angle equal to $90^{\circ}$, the inducer creates EC flow along the $z$ axis, which advances the detection of the $B_{x}$ component regardless of the orientation of the crack (cases 1-1 and 2-1). When $\alpha=0^{\circ}$, the inducer generates EC flow parallel to the $x$ axis and thus promotes the $B_{z}$ field detection for both orientations of the crack (cases 1-3 and 2-3). Therefore, the measurement of a single component configuration $\left(B_{x}\right.$ or $\left.B_{z}\right)$ for the crack detection cannot be used to estimate the orientation of the observed crack. To determine the crack orientation, we propose, in the next section, to measure simultaneously the two tangential magnetic flux density $B_{x}$ and $B_{z}$ along the $x$ - and $z$-axis, respectively.

\section{Tangential magnetic configuration detection}

To add the ability of the presented NDT system to determine the crack orientation, we examine the tangential magnetic flux density, $B_{x z}$, resulting from the components $B_{\mathrm{X}}$ and $B_{\mathrm{Z}}$ with

$B_{x z}=B_{x}+B_{z}$.

Fig. 5 shows the variation of the tangential magnetic flux density, $\left|\Delta B_{x z}\right|$, versus the relative position $x$ of the system and for different values of the excitation angle $\alpha$. Three orientations of the crack were studied $\left(\beta=0^{\circ}, 90^{\circ}\right.$ and $\left.45^{\circ}\right)$. For reasons of symmetry, we studied $\alpha$ only over the range from $0^{\circ}$ to $180^{\circ}$.

The variations of the signal amplitude, $\left|\Delta B_{x z}\right|$, with the excitation angle $\alpha$ provide information about the orientation and the position of the observed crack. Indeed, the signal, $\left|\Delta B_{x z}\right|$, in the 

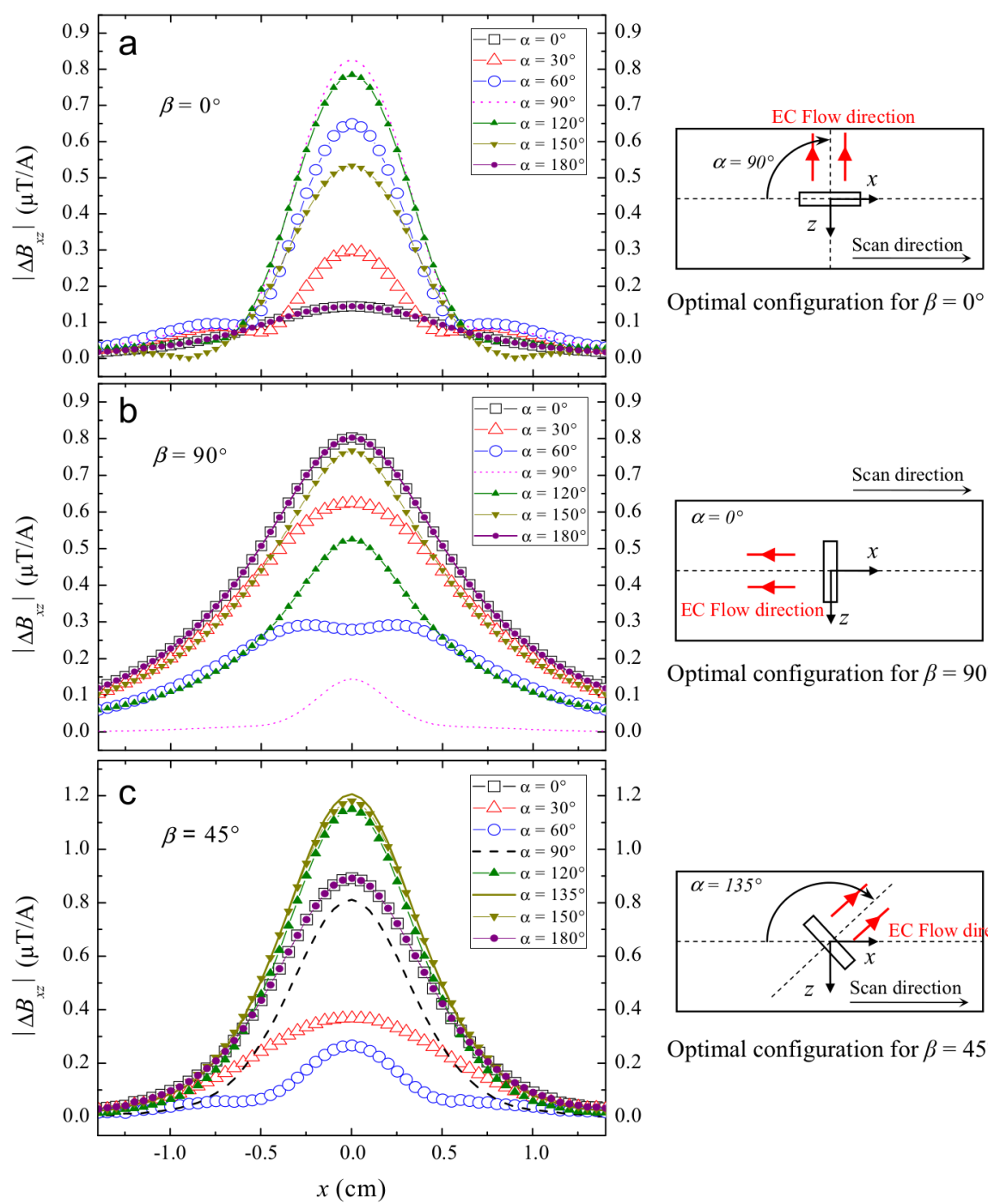

Optimal configuration for $\beta=0^{\circ}$

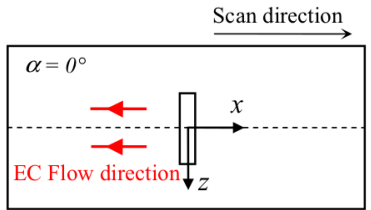

Optimal configuration for $\beta=90^{\circ}$

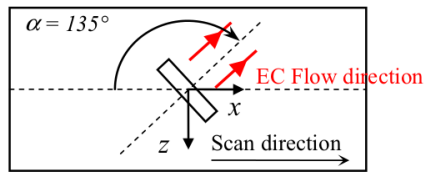

Optimal configuration for $\beta=45^{\circ}$

Fig. 5. Numerical magnetic flux density valuation, $\mid \Delta B_{x} \neq$, versus the relative system position, $x$, for different values of $\alpha$ and for three orientations of the crack: (a) $\beta=90^{\circ}$, (b) $\beta=0^{\circ}$, (c) $\beta=45^{\circ}$. ( $h_{\text {inducer }}=1 \mathrm{~mm}, h_{\mathrm{GMR}}=0.5 \mathrm{~mm}, L_{\text {crack }}=8 \mathrm{~mm}, h_{\text {crack }}=8 \mathrm{~mm}, w_{\text {crack }}=0.1 \mathrm{~mm}, \sigma \approx 1.68 \times 10^{7} \mathrm{~S} / \mathrm{m}, f_{\text {exc }}=400 \mathrm{~Hz}$ ).

a

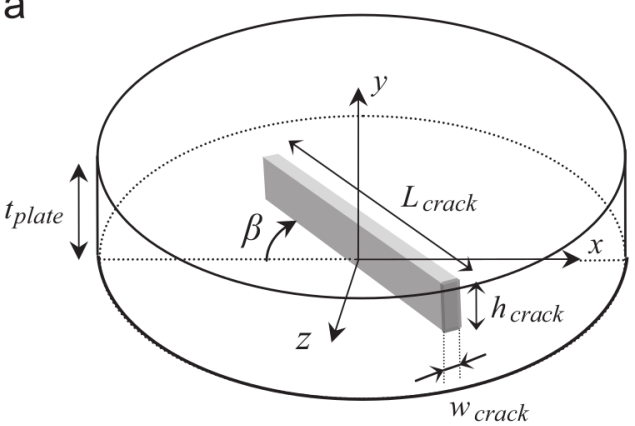

b

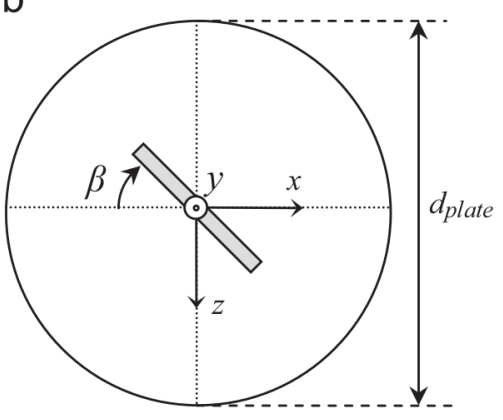

Fig. 6. 3D sketch view (a) and top view (b) of the geometry of the aluminum wedge used for the comparison between experimental to theoretical results $\left(d_{\text {plate }}=6 \mathrm{~cm}\right.$, $\left.t_{\text {plate }}=1 \mathrm{~cm}, L_{\text {crack }}=8 \mathrm{~mm}, h_{\text {crack }}=8 \mathrm{~mm}, w_{\text {crack }}=0.5 \mathrm{~mm}, \sigma \approx 1.97 \times 10^{7} \mathrm{~S} / \mathrm{m}, \beta=45^{\circ}\right)$.

three cases, is a maximum for a given value of $\alpha$ that induces currents perpendicular to the crack direction. The knowledge of the excitation angle optimizing the signal amplitude determines the direction of the crack. For example, by analyzing the Fig. $5 c$, we know that the orientation of the crack is $45^{\circ}$. Indeed, the signal $\left|\Delta B_{x z}\right|$ is maximal for $\alpha$ equal to $135^{\circ}$. Moreover, the position where the measured signal amplitude is maximum determines the $(x, z)$ coordinate of the crack.

\section{Experimental validation}

Experimental and numerical results were compared in the case of the inspection of an aluminum disk with a single centered crack. The crack makes an angle $\beta=45^{\circ}$ with the scanning direction along the $x$ axis (Fig. 6).

The experimental measurements were performed for two orientations of a single inducer wire along the $x$ - and $z$-axis. These 
a
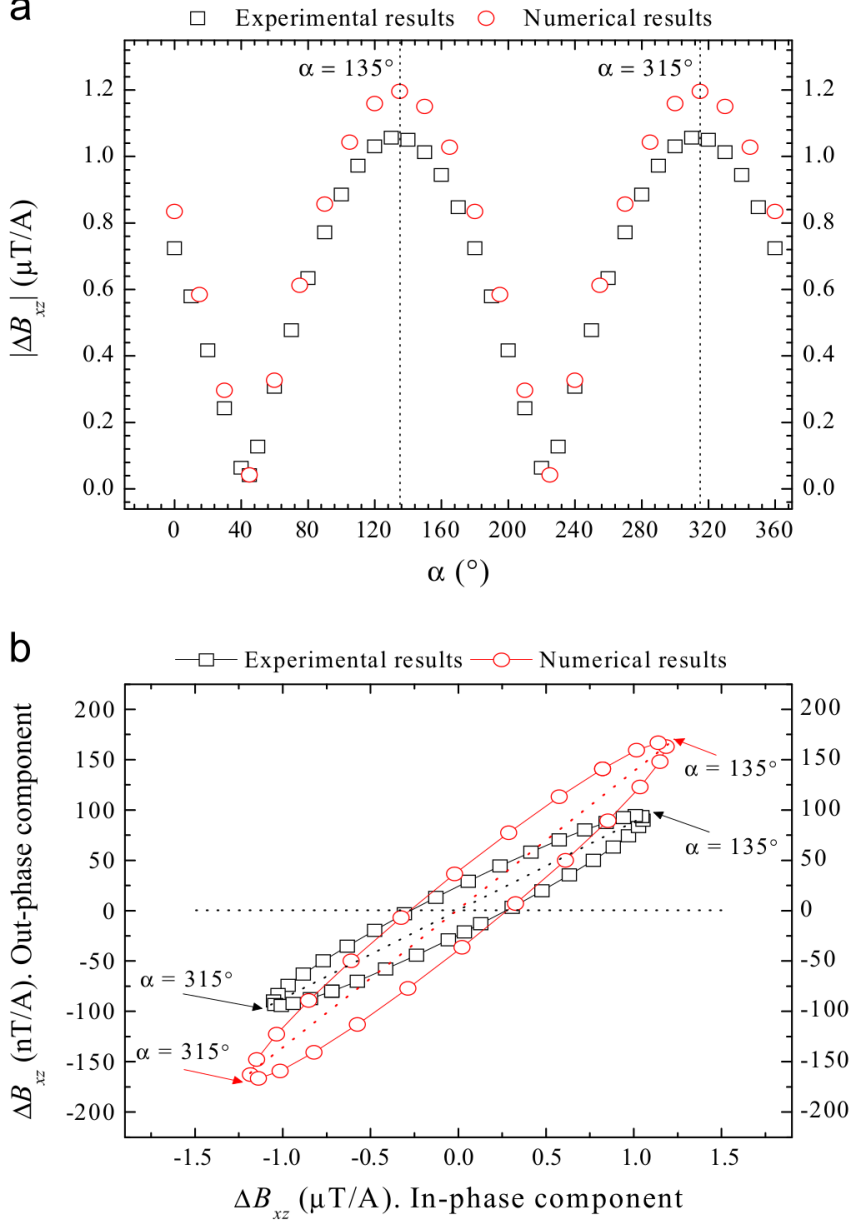

Fig. 7. Comparison of the sensed magnetic field, $\Delta B_{x z}$, between the simulation and measurements (a) modulus of the tangential magnetic flux density $\left|\Delta B_{x z}\right|$, (b) $\Delta B_{x z}$ Lissajous curves. $\left(x=0 \mathrm{~mm}, \beta=45^{\circ}, h_{\text {inducer }}=1 \mathrm{~mm}, h_{G M R}=2 \mathrm{~mm}, L_{\text {crack }}=8 \mathrm{~mm}\right.$, $\left.h_{\text {crack }}=8 \mathrm{~mm}, w_{\text {crack }}=0.5 \mathrm{~mm}, \sigma=1.97 \times 10^{7} \mathrm{~S} / \mathrm{m}, f_{\text {exc }}=1 \mathrm{kHz}\right)$.

configurations correspond to the rotating magnetic field inducer with the values of the excitation angle $\alpha$ equal to $0^{\circ}$ and $90^{\circ}$. To sense the tangential magnetic flux density $B_{x z}$, the sensing axis of the IGMR magnetometer used was set in $x$-and $z$-axis for each orientation of the inducer $\left(\alpha=0^{\circ}\right.$ and $\left.90^{\circ}\right)$. We assumed that the tangential magnetic flux density signal $\Delta B_{x z}$, in function of excitation angle $\alpha$, is given by the superposition of the different measurements, as

$\Delta B_{x z}(\alpha)=\cos (\alpha) \Delta B_{x z}\left(\alpha=0^{\circ}\right)+\sin (\alpha) \Delta B_{x z}\left(\alpha=90^{\circ}\right)$.

The measurements were performed with an inducer current frequency of $1 \mathrm{kHz}$ and amplitude of $84 \mathrm{~mA}$. The lift-offs of the inducer and the IGMRM over the plate were 1 and $2 \mathrm{~mm}$, respectively. The experimental setup used in this paper was described in detail in previous papers $[6,10]$. The shape of the signal $\left|\Delta B_{x z}\right|$, and the Lissajous curves are plotted as a function of the excitation angle $\alpha$ at the position $(x=0)$ where the signal $\left|\Delta B_{x z}\right|$ reaches its maximum. The experimental and simulation results for different $\alpha$ values between $0^{\circ}$ and $360^{\circ}$ are plotted in Fig. 7 .
The amplitude and phase of $\Delta B_{x z}$, which are deduced from the measurements, are in good agreement with the simulation. The slight differences between the experiment and the model can be explained by the uncertainties between the real parameters of the system and those used in the simulation, such as the lift-off and the relative position of the sensor and inducer over the plate under test.

In Fig. 7, the curves reached a maximum for the values of the excitation angle $\alpha$ that induced currents perpendicularly to the crack direction. In this manner, we are able to simply characterize the crack orientation because of the dependence of $\Delta B_{x z}$ with the direction of the current.

\section{Conclusion}

In this study, we proposed a method for the detection and characterization of deep cracks. This method uses an original excitation inducer to generate a pseudo-rotating magnetic field. By using this type of method or inducer, we can easily determine the crack direction by analyzing the sensed magnetic field signal due to the crack versus the EC and the induction excitation angle $\alpha$. Only a simple amplitude or Lissajous curve provides the information. This method is an alternative to the ones that use complex inverse methods for crack characterization. The results presented in this paper are promising, and further developments involve building a system that simultaneously measures the magnetic flux density in the two tangential directions and automatically control the excitation angle of the currents in the inducer.

\section{References}

[1] Yang Guang, Tamburrino Antonello, Udpa Lalita, Udpa Satish S, Zeng Zhiwei, Deng Yiming, et al. Pulsed eddy-current based giant magnetoresistive system for the inspection of aircraft structures. IEEE Transactions on Magnetics 2010:46(3):910-7.

[2] Vacher F, Gilles-Pascaud C, Decitre JM, Fermon C, Pannetier M, Cattiaux G. Non destructive testing with gmr magnetic sensor arrays. In: Proceedings of the 9th ECNDT. Berlin; 2006.

[3] Hamia R, Cordier C, Saez S, Dolabdjian C. giant magneto impedance sensor for non destructive evaluation eddy current system. Sensor Letter 2009;7 (3):437-41.

[4] Hamia R, Cordier C, Saez S, Dolabdjian C. Eddy current non destructive testing using an improved giant magnetoresistance magnetometer and a single wire as inducer.a benchmark performance analysis with the help of the finite element method. IEEE Transactions on Magnetics 2010:46(10):3731-7.

[5] Yang G, Zeng Z, Deng Y, Liu X, Udpa L, Tamburrino A, et al. 3D EC-GMR sensor system for detection of subsurface defects at steel fastener sites. NDT and E International 2012;50:20-8.

[6] Dolabdjian C, Waché G, Perez L. Improvement in subsurface fatigue cracks under airframes fasteners detection using improved rotary Giant MagnetoResistance Magnetometer head. Insight-Non-Destructive Testing and Condition Monitoring 2007:49(2):133-6.

[7] Ma YP, Wikswo P. SQUID magnetometers for depth-selective, oriented eddy current imaging. Review of Progress in QNDE 1998;17A:1067-74.

[8] Perez L, Le hir J, Dolabdjian C, Butin L. Investigation in detection of fatigue cracks under rivet head airframe using improved GMR magnetometer in an Eddy Current system. Journal of Electrical Engineering 2004;55(10/S):10-1.

[9] Perez L, Dolabdjian C, Waché G, Butin L. Advance in magnetoresistance magnetometer performances applied in eddy current sensor arrays. In: Proceedings of the 16th WCNDT'04 Conference.Montréal; 28 August-03 September 2004

[10] Hamia R, Cordier C, Saez S, Dolabdjian C. Separability of multiple deep crack defects with an NDE eddy current system. IEEE Transactions on Magnetics $2013 ; 49(1): 124-7$ 